Manuscript Number: CJC-D-19-00379R1

Title: Acute Myocardial Infarction in Severe Mental Health Illness: prevalence, clinical outcomes and process of care in United States hospitalizations.

Article Type: Original Research Clinical

Corresponding Author: Dr. Mohamed Osama MOHAMED, MBBCh, MRCP(UK)

Corresponding Author's Institution: Keele Cardiovascular Research Group

First Author: Mohamed Osama MOHAMED, MBBCh, MRCP(UK)

Order of Authors: Mohamed Osama MOHAMED, MBBCh, MRCP(UK); Muhammad Rashid; Saeed Farooq; Nishat Siddiqui; Purvi Parwani; David Shiers; Ritu Thamman; Martha Gulati; Ahmad Shoaib; Carolyn Chew-Graham; Mamas A Mamas

Abstract: Background: Severe mental illness (SMI) is associated with increased cardiovascular mortality. We sought to examine the prevalence, clinical outcomes and management strategy of patients with SMI presenting with acute myocardial infarction (AMI).

Methods: All AMI hospitalizations from the National Inpatient Sample were included, stratified by mental health status in to 5 groups: no-SMI, Schizophrenia, 'Other non-organic psychoses' (ONOP), Bipolar Disorder and Major Depression. Regression analyses were performed to assess the association (adjusted odds ratios, $\mathrm{p} \leq 0.001$ for all outcomes) between SMI subtypes and clinical outcomes.

Results: Out of 6,968,777 AMI hospitalizations between 2004 and 2014, 439,544 (6.5\%) had an SMI diagnosis. Although patients with Schizophrenia and ONOP experienced higher crude rates of in-hospital mortality and stroke compared to those without SMI, only Schizophrenia patients were at increased odds of mortality $(1.10[1.04,1.16])$, while ONOP were the only group at increased odds of stroke $(1.53[1.42,1.65])$ following multivariate adjustment. Patients with ONOP were the only group associated with increased odds of in-hospital bleeding compared to those without SMI (1.11[1.04,1.17]). All SMI subtypes were less likely to receive coronary angiography and PCI, with the Schizophrenia group being at least odds of either procedure $(0.46[0.45,0.48]$ and $0.57[0.55,0.59]$, respectively).

Conclusion: Schizophrenia and 'other non-organic psychoses' are the only SMI subtypes associated with adverse clinical outcomes after AMI. However, all patients with SMI were less likely to receive invasive management for AMI, with women and schizophrenia diagnosis being the strongest predictors of conservative management. A multidisciplinary approach between psychiatrists and cardiologists could improve outcomes of this high-risk population. 
Dear Professor Stanley Nattel, MD, Editor in Chief,

We thank the Editorial Committee and the Reviewers for their valuable comments on the manuscript entitled 'Acute Myocardial Infarction in Severe Mental Health Illness: prevalence, clinical outcomes and process of care in United States hospitalizations.' and feel that these recommendations have improved the quality of our manuscript. We have attempted to answer all the comments fully as outlined in the rebuttal and tracked all new changes in the manuscript. Furthermore, we acknowledge all the recommendations by the Editor-in-Chief and Editorial manager and can confirm that all the requested changes have been made including reducing the word count to 5600 words.

We hope that we have addressed all reviewers' and editorial manager's comments sufficiently and hope that these changes will enable publication of our paper in the Canadian Journal of Cardiology.

As per your request we can confirm the following:

1. No portion of the text has been copied from other material in the literature (unless in quotation marks, with citation).

2. We are aware that it is the author's responsibility to obtain permission for any figures or tables reproduced from any prior publications, and to cover fully any costs involved. Such permission must be obtained prior to final acceptance. However, we have not reproduced any figures or tables from prior publications.

We list our reply to the reviewer's comments in the file entitled 'Response to Reviewers' as per journal instructions.

Yours sincerely

Mohamed Mohamed and Mamas Mamas

On behalf of submitting authors 


\section{Responses to Reviewers}

\section{Reviewer \#1:}

Comment 1. It would be very interesting and important for the readers of CJC to see an analysis looking at temporal trends of outcomes from 2004 to 2014.

Response: We thank the reviewer for kindly highlighting this important aspect of the analysis. We have performed a temporal analysis of clinical outcomes and receipt of invasive management and have provided the data in Table S5 (clinical outcomes) and Figure S2 (growth of coronary angiography and PCI). Furthermore, we have updated the 'Results' sections with the respective findings (quoted below).

Under 'Utilization of invasive management' subheading:

"There was a notable increase in the rates of both coronary angiography and PCI in all the groups over the study period (Figure $S 2$ )."

Under 'In-hospital MACCE, Mortality and Stroke' subheading:

"A temporal analysis of outcomes demonstrates an overall decline in the rates of MACCE, mortality and acute stroke/TIA in all the study groups except the ONOP and Bipolar disorder groups whose rates of MACCE and acute stroke/TIA have either increased or remained the same (Table S5). However, the pattern of differences in rates of MACCE, morality and stroke between groups remained the same throughout the study period."

Under 'In-hospital Bleeding' subheading:

"The rate of all-cause bleeding increased in all groups over the study period but remained higher in SMI groups compared to patients without SMI, with the exception of ONOP group whose rates of bleeding were higher than patients without SMI. (Table S5)"

Comment 2. In addition, please provide an analysis looking at the differences in outcomes between NSTE-ACS and STEMI.

Response: We have performed an analysis of outcomes according to the type of MI (STEMI vs. NSTEMI) and presented the findings in Table S6. Furthermore, we have created a dedicated subheading in the 'Results' section to report our findings (quoted below).

\section{"Sensitivity Analysis}

A sensitivity analysis according to AMI type (STEMI vs. NSTEMI) was performed to exclude examine differences in outcomes between both AMI presentations. Overall, the findings in the STEMI and NSTEMI groups were generally similar to those found in the total cohort. (Table S6) However, certain differences were observed within AMI subgroups. In the NSTEMI subgroup, patients with Bipolar Disorder were associated with reduced odds of MACCE compared to those without SMI while there was no difference in MACCE and all-cause bleeding between patients without SMI and those with ONOP. In the STEMI subgroup, there was no difference in mortality and all-cause bleeding between patients with Bipolar Disorder group and those without SMI as was the case in all-cause bleeding between patients with Major Depression and those without SMI." 
Comment 3. It would be worth to run a multivariable analysis according to gender rather than just presenting crude rates.

Response: We thank the reviewer for this suggestion. We have conducted a multivariable analysis according to gender with male gender being the reference group. The findings are summarized in Table S7 and the 'Results section' has been updated accordingly (quoted below).

Under 'Utilization of invasive management' subheading:

"The rates of coronary angiography and PCI were also lower amongst women across all study groups, including patients without an SMI diagnosis (Figure 3), a finding that persisted in multivariate analysis (Table S7). However, the odds of receipt of coronary angiography between genders were not significant in the Schizophrenia and Major Depression groups. “

Under 'In-hospital MACCE, Mortality and Stroke' subheading:

"In multivariable analysis, the odds of MACCE were increased in women without SMI and those with Bipolar Disorder and Major Depression, primarily driven by increased odds of acute stroke in these groups. (Table S7) In contrast to the other groups, women with ONOP were associated with reduced odds of MACCE and stroke compared to men (Table S7). Furthermore, women without SMI and those with ONOP and Major Depression were at reduced odds of mortality compared to men, while there was no difference in mortality between genders in the Schizophrenia and Bipolar Disorder groups.",

Under 'In-hospital Bleeding' subheading:

"A multivariate analysis according to gender revealed increased odds of bleeding in women without SMI as well as those with Schizophrenia and Major depression (Supplementary Material - Table S7). In contrast, no difference in bleeding was found between genders in patients with ONOP and women with Bipolar disorder were at reduced odds of bleeding."

Furthermore, we have updated the relevant section in the 'Discussion' section, which now reads as follows:

"We found women in all study group to be associated with lower or insignificant odds of mortality compared to men, but also at increased odds of acute stoke/TIA, except in those with ONOP where the was no difference between genders. Women with Schizophrenia were also associated with the highest odds of bleeding complications compared to men. However, women of all subgroups were much less likely to undergo invasive management compared to men, especially women with Bipolar Disorder who were $15 \%$ and $34 \%$ less likely to receive coronary angiography and PCI, respectively."

Comment 4. I liked your figures in Forest plots, so, could you illustrate the aOR of PCI and coronary angiography as well?

Response: We are grateful for the reviewers' kind remarks. Figure 6 now includes forest plots of aOR for receipt of coronary angiography and PCI. The figure is also attached below: 


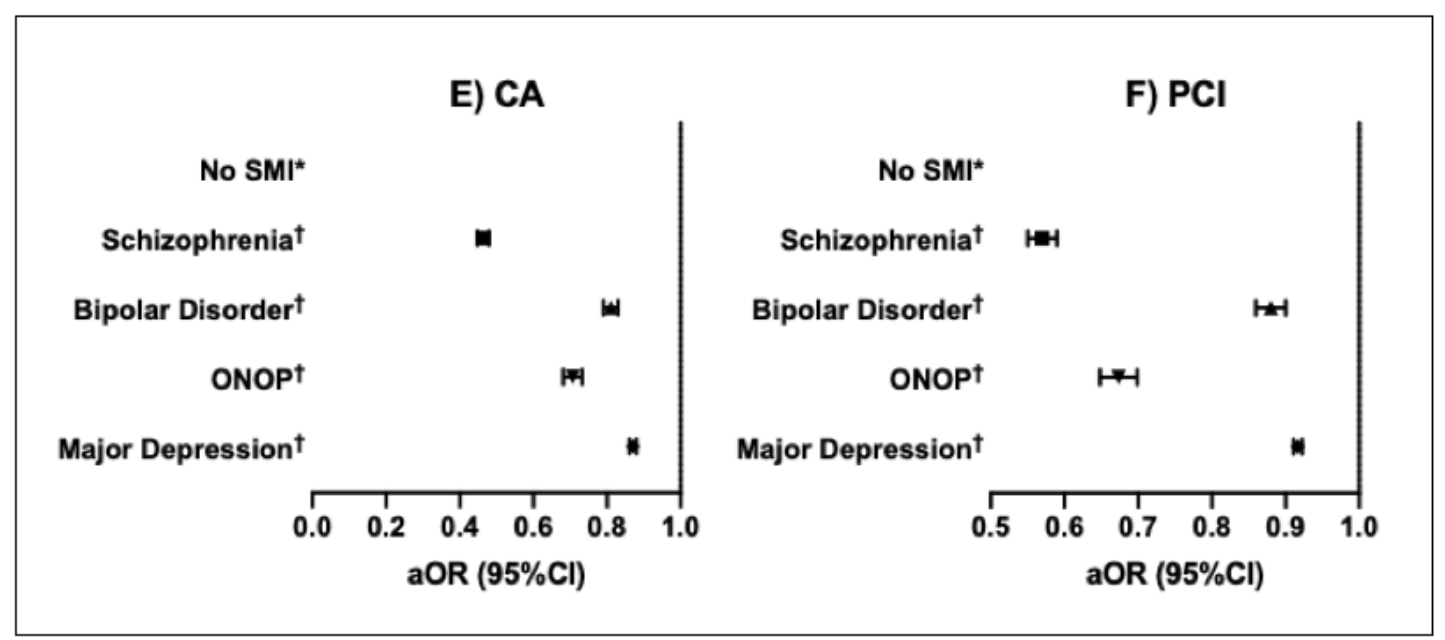

*Reference group is No-SMI; $\S$ non-significant $(\mathrm{p} \geq 0.05) ; \dagger \mathrm{p}<0.001$; CI: confidence interval; ONOP: Other non-organic psychoses; SMI: Severe mental illness

Comment 5. Please maintain consistency in US or UK spelling. I noticed that the tables are spelt in British English and the manuscript is in American English with random words e.g. 'centre' and 'analysed' in British English.

Response: We thank the reviewer for highlighting this issue. This issue has now been rectified and all the text has been modified to US English spelling. 


\section{Reviewer \#2:}

Comment 1. The second and third sentences of the Results section of the Abstract sound partly contradictory and need to be read more than once to be understood: "Patients with Schizophrenia and ONOP experienced higher crude rates of in-hospital mortality and stroke compared to those without SMI. However, only Schizophrenia was associated with increased odds of mortality (aOR: 1.10[1.04,1.16]) and only ONOP were associated with increased odds of stroke (aOR: 1.53[1.42,1.65])." Consider rewriting this to make it clearer.

Response: We thank the reviewer for highlighting the lack of clarity of the aforementioned statement and we can confirm that this has now been changed to further clarify its intended meaning. The new statement reads as follows (also highlighted in the manuscript):

"Although patients with Schizophrenia and ONOP experienced higher crude rates of inhospital mortality and stroke compared to those without SMI, only Schizophrenia patients were at increased odds of mortality $(1.10[1.04,1.16])$, while ONOP were the only group at increased odds of stroke (1.53[1.42,1.65]) following multivariate adjustment."

Comment 2. Consider briefly speculating in the Discussion on why the proportion of MI patients with major depression has increased so dramatically over the study period. Has major depression become more common on the population at risk for MI? Is major depression being coded more often, with less real change in prevalence?

Response: Thank you for highlighting this important point that is lacking in the discussion. There is data from several nationwide studies to suggest that the prevalence of both major depression and bipolar disorder has increased in the background population (references below). Nevertheless, we agree with the reviewer that the increase in prevalence may be due to major depression being coded more frequently. We have added a paragraph to the discussion to emphasize both possibilities to readers. The paragraph reads as follows:

"The present study is the first to examine the prevalence of SMI in the AMI population and shows a rise in the prevalence of SMI over an eleven-year horizon, primarily due increasing rates of Major Depression and Bipolar disorder diagnoses. Although these findings are in keeping with previous reports of trends in the general population, it is possible that that these diagnoses have been coded more frequently in later years due to physicians' awareness of their implications in the context of AMI hospitalizations"

\section{References:}

- Sara GE, Malhi GS. Trends in diagnosis of bipolar disorder: Have the boundaries changed? Aust. N. Z. J. Psychiatry. 2015;49:1021-1028.

- Weinberger AH, Gbedemah M, Martinez AM, Nash D, Galea S, Goodwin RD. Trends in depression prevalence in the USA from 2005 to 2015: widening disparities in vulnerable groups. Psychol. Med. 2018;48:1308-1315.

- Jeuring HW, Comijs HC, Deeg DJH, Stek ML, Huisman M, Beekman ATF. Secular trends in the prevalence of major and subthreshold depression among 55-64-year olds over 20 years. Psychol. Med. 2018;48:1824-1834. 
Comment 3. How accurate are co-morbidities coded, specifically ones such as dyslipidemia and family history of coronary disease?

Response: As with most administrative datasets, an inherent limitation is the possible coding inaccuracy and the reliance on data that has been collected in retrospect. However, we have stated this as our first limitation in the manuscript's 'limitations' section, "The are several limitations to the present study. First, the NIS is an administrative dataset that is susceptible to coding. Although the identification of ACS and SMI diagnoses as well as other comorbidities was based on the use of administrative codes, ICD-9 codes have been previously validated for the purposes of cardiovascular research.",

References:

- Birman-Deych E et al. Accuracy of ICD-9-CM codes for identifying cardiovascular and stroke risk factors. Med Care. 2005. May;43(5):480-5.

- DeShazo et al. A comparison of a multistate inpatient EHR database to the HCUP Nationwide Inpatient Sample; BMC Health Services Research (2015) 15:384 DOI $10.1186 / \mathrm{s} 12913-015-1025-7)$

More specifically, the NIS has been previously validated for the purpose of cardiovascular research in regard to its demographic capture. We have clarified this in our 'Methods' section under the 'Data source' subheading (now in Supplementary Material - Appendix B to reduce the word count based on the Editorial Office's request):

'The estimates of hospital characteristics, numbers of discharges, length of stay, and inhospital mortality from the HCUP Nationwide Inpatient Sample (NIS) for 2007 were highly comparable to three related data sources: the American Hospital Association (AHA) Annual Survey Database, the National Hospital Discharge Survey (NHDS) from the National Center for Health Statistics, and the MedPAR inpatient data from the Centers for Medicare and Medicaid Services (CMS). Furthermore, NIS was found to have a more comprehensive demographic capture when compared to a large multistate EHR dataset in more than 25 diagnosis groups, including cardiovascular disorders'

References:

- Barrett M WE, Whalen D. HCUP Nationwide Inpatient Sample (NIS) Comparison Report. HCUP Methods Series Report. 2007.

- DeShazo JP, Hoffman MA. A comparison of a multistate inpatient EHR database to the HCUP Nationwide Inpatient Sample. BMC health services research. 2015;15:384.

Comment 4. The authors state (page 4) that SMI patients are under-represented in clinical trials. It might be more accurate and comprehensive to state that SMI is usually an exclusion factor in cardiovascular trials, or SMI patients are excluded because they are unable to reliably complete follow-up and comply with treatment.

Response: We thank the reviewer for their suggestion and completely agree with that a rephrase of the statement to elaborate why SMI patients are under-represented in clinical trials would be more insightful. The aforementioned sentence has now been changed to the following:

"Acute myocardial infarction (AMI) is the commonest manifestation of CVD but there is limited literature on the prevalence, treatment strategies and clinical outcomes of patients 
with SMI who are often excluded from clinical trials due to concerns about their adherence to cardiovascular medications and follow-up."

Comment 5. The authors note that psychotropic drugs, such as SSRI's, used by SMI patients have cardiovascular effects. Unfortunately, concomitant medications are not included in their database. Consider adding a bit more detail on how these drugs might promote MI or interfere with antiplatelet drugs, since cardiologists need to be aware of this, even though it is not directly related to the results of this study.

Response: We have conducted a search on the association between psychotropic medications and MI and, while there is a clear association between the two, the evidence regarding pathophysiological mechanisms by which psychotropic medications could promote MI is sparse. We have now expanded the second paragraph of the 'Discussion' section to inform readers about the currently hypothesized mechanisms. The relevant addition reads as follows:

"Antipsychotics and antidepressants such as SSRI's (selective serotonin reuptake inhibitors) have been shown to increase the risk of metabolic syndrome due to their interaction with target 5-HT neuroreceptors that modulate weight control and glucose metabolism, which increases the risk of myocardial infarction. Furthermore, the use of antipsychotics (typical and atypical) has been associated with an increased risk of cerebrovascular events, especially in elderly patients, although the underlying mechanism remains unknown, while SSRI's are thought to increase the uptake of serotonin into platelets leading to increased bleeding tendency."

Comment 6. In the SWEDEHEART Registry, the odds ratio for 30-day mortality in schizophrenia was 2.58 (1.88-3.54), much higher than the $10 \%$ increased risk that the authors report. Is it possible that the SWEDEHEART data is more relevant because follow-up includes hospital discharge to 30 days?

Response: We thank the reviewer for highlighting this important observation. We believe that there are several reasons for the differences in risk or odds of mortality between our study and those reported in the SWEDEHEART study. First, the SWEDEHEART registry included a very small number of patients compared to our study (SWEDEHEART vs. present study: Bipolar $n=442$ vs. 41,362 ; Schizophrenia $n=541$ vs. 23,582 ) and these patients' demographics and presentation were different to those found in our cohort, which makes it difficult to compare both studies. For example, the SWEDEHEART study only included patients with a first presentation of MI and had a significantly higher proportion of STEMI presentation amongst Schizophrenia and Bipolar patients. Furthermore, patients with Schizophrenia and Bipolar disorder in their analyses were significantly older with a higher prevalence of risk factors such as heart failure and previous stroke compared to our study. The baseline differences between both studies is likely attributable to their sample size rather than geographical differences and may have inflated their risk of mortality amongst both Schizophrenia and Bipolar disorder patients. However, we do agree that the risk of mortality may become more pronounced with longer follow up and we had already stated this in our 'Limitations section' (quoted below).

"Second, the ischemic benefits of an invasive strategy over a conservative management of AMI have been primarily shown in studies reporting at least 30-day follow 
up. Therefore, it is possible that SMI patients experience worse clinical outcomes in the long term than those reported in the present study that only captures in-hospital outcomes."

Comment 7. Figure 1A is unnecessary and could be deleted.

Response: We agree with the reviewer that illustrating the prevalence of all-cause SMI is less useful compared to individual SMI subtypes and we have now removed this figure. Figure 1 now includes the prevalence of SMI from 2004 to 2014 according to subtype (previously Figure 1B).

Comment 8. The authors refer to their study population as "contemporary" at several points in the paper. Perhaps 2004-2014 is not contemporary any more, but this is a minor quibble.

Response: At the time of analysis, the latest available dataset was for 2016 as there is always a two-year lag in NIS data being sold for research use. We had not included the latter two years for two reasons. First, they were not accessible for our use. Second, the transition to ICD-10 diagnosis codes started in 2015 and, in view of the significant differences in the coding system, especially for mental health disorders, we felt that this may introduce a potential selection bias when combined with ICD-9 diagnoses in our analysis.

However, we are in complete agreement with the reviewer in that the word 'contemporary' may not be accurate and we have removed all references to it from our manuscript. 


\section{Acute Myocardial Infarction in Severe Mental Health Illness: prevalence, clinical outcomes and process of care in United States hospitalizations.}

Short title: Effect of severe mental illness on AMI outcomes.

Mohamed Osama Mohamed ${ }^{1}, \mathrm{MB}$ BCh, MRCP(UK), Muhammad Rashid ${ }^{1}$, MBBS, MRCP(UK), Saeed Farooq ${ }^{2,3}$, PhD, FCPS(Psych), Nishat Siddiqui ${ }^{4}$, MB BCh, MRCP (UK), Purvi Parwani $^{5}$, MD, David Shiers ${ }^{6}$, MB ChB, MRCP(UK), MRCGP, Ritu Thamman ${ }^{7}$, MD, Martha Gulati $^{8}$, MD, Ahmad Shoaib ${ }^{1}$, MD, Carolyn Chew-Graham ${ }^{2,3}$, BSc, MB ChB, MD, FRCGP, Mamas A. Mamas ${ }^{1}$, MA, MRCP, DPhil

(1) Keele Cardiovascular Research Group, Centre for Prognosis Research, Institute of Applied Clinical Science and Primary Care and Health Sciences, Keele University, UK

(2) Research Institute for Primary Care and Health Sciences, Keele University, UK

(3) Midlands Partnership NHS Foundation Trust, Staffordshire, UK

(4) Nevill Hall Hospital, Aneurin Bevan University Health Board, Wales, UK

(5) Division of Cardiology, Department of Medicine, Loma Linda University Health, Loma Linda, California

(6) Psychosis Research Unit, Greater Manchester Mental Health NHS Foundation Trust, UK

(7) Department of Cardiology, University of Pittsburgh, PA, USA

(8) Division of Cardiology, University of Arizona, Phoenix, AZ, USA

\section{Correspondence to:}

Mamas A. Mamas

Professor of Cardiology

Keele Cardiovascular Research Group,

Centre for Prognosis Research,

Institute for Primary Care and Health Sciences,

Keele University, UK

mamasmamas1@yahoo.co.uk

Key Words: Severe Mental Illness, Bipolar Disorder, Schizophrenia, Major Depression, Psychosis, Acute Myocardial Infarction, outcomes, management 


\section{Abbreviations}

ACS

AHRQ

AMI

CA

CABG

CAD

CVA

DAPT

MACCE

NST-EACS

OR

PCI

RR

SMI

STEMI
Acute Coronary Syndrome

Agency for Healthcare Research and Quality

Acute Myocardial Infarction

Coronary Angiography

Coronary Artery Bypass Grafting

Coronary Artery Disease

Cerebrovascular Accident

Dual Anti-Platelet Therapy

Major Adverse Cardiovascular and Cerebrovascular

Event

Non-ST-elevation acute coronary syndrome

Odds Ratio

Percutaneous Coronary Intervention

Relative Risk or Risk Ratio

Severe Mental Illness(es)

ST-elevation Myocardial Infarction 


\section{Abstract}

Background: Severe mental illness (SMI) is associated with increased cardiovascular mortality. We sought to examine the prevalence, clinical outcomes and management strategy of patients with SMI presenting with acute myocardial infarction (AMI).

Methods: All AMI hospitalizations from the National Inpatient Sample were included, stratified by mental health status in to 5 groups: no-SMI, Schizophrenia, 'Other non-organic psychoses' (ONOP), Bipolar Disorder and Major Depression. Regression analyses were performed to assess the association (adjusted odds ratios, $\mathrm{p} \leq 0.001$ for all outcomes) between SMI subtypes and clinical outcomes.

Results: Out of 6,968,777 AMI hospitalizations between 2004 and 2014, 439,544 (6.5\%) had an SMI diagnosis. Although patients with Schizophrenia and ONOP experienced higher crude rates of in-hospital mortality and stroke compared to those without SMI, only Schizophrenia patients were at increased odds of mortality $(1.10[1.04,1.16])$, while ONOP were the only group at increased odds of stroke $(1.53[1.42,1.65])$ following multivariate adjustment. Patients with ONOP were the only group associated with increased odds of inhospital bleeding compared to those without SMI $(1.11[1.04,1.17])$. All SMI subtypes were less likely to receive coronary angiography and PCI, with the Schizophrenia group being at least odds of either procedure $(0.46[0.45,0.48]$ and $0.57[0.55,0.59]$, respectively).

Conclusion: Schizophrenia and 'other non-organic psychoses' are the only SMI subtypes associated with adverse clinical outcomes after AMI. However, all patients with SMI were less likely to receive invasive management for AMI, with women and schizophrenia diagnosis being the strongest predictors of conservative management. A multidisciplinary approach between psychiatrists and cardiologists could improve outcomes of this high-risk population. 


\section{Condensed Abstract (<75 Words)}

Patients with severe mental illness (SMI) are a high-risk group for cardiovascular disease. The present study examined the prevalence and outcomes of SMI patients in a national AMI cohort. SMI has doubled in prevalence amongst AMI patients, mainly due to a rise in Major Depression and Bipolar Disorder. Only Schizophrenia and 'Other non-organic psychoses' groups were associated with worse outcomes, but all SMI subtypes were less likely to receive invasive management for their AMI.

\section{Introduction}

Cardiovascular disease (CVD) is a major cause of morbidity and mortality in patients with severe mental illness (SMI), including Schizophrenia, Bipolar Disorder Major Depression and Psychosis and Delusional disorders. $\frac{1-4}{}$ It is estimated that more than 10.4 million adults (4.2\% of all adults) suffer from SMI in the United States, with a similar worldwide prevalence of up to $6.8 \% . \underline{5} \underline{6}$

Patients with SMI are reported to have a two- to three-fold increased risk of mortality from cardiovascular disorders compared to the general population, a risk that has increased over the past two decades. 1 Acute myocardial infarction (AMI) is the commonest manifestation of CVD but there is limited literature on the prevalence, treatment strategies and clinical outcomes of patients with SMI who are often excluded from clinical trials due to concerns about their adherence to cardiovascular medications and follow-up. $\underline{8}$ A nationwide analysis in patients with Schizophrenia and Bipolar Disorder presenting with ST-Elevation Myocardial Infarction (STEMI) demonstrated an increased mortality in patients with Schizophrenia and reduced odds of revascularization in both subgroups. $\underline{9}$ Conversely, an analysis of elderly patients (>65 years) presenting with AMI showed that patients with mental 
health disorders (Schizophrenia, affective disorders and substance abuse disorder) were not at increased risk of 30-day mortality, although they were also less likely to undergo coronary revascularization. $\underline{10}$

While previous studies have looked at the clinical outcomes of SMI in the context of AMI, they have demonstrated conflicting results and were subject to limitations such as analysis of selected cohorts (single ACS subtype, e.g. STEMI) $\underline{9}$ or a single geographical region (e.g. State of Florida) $\underline{11}$, inclusion of selected outcomes (e.g. only mortality) $\underline{12}$, exclusion of patients with previous myocardial infarction $\underline{12}$, or the analysis of small sample that may not be representative of the population of interest $\underline{13}$. Furthermore, patients with SMI are a heterogenous population with differences in their underlying pathophysiology and pharmacotherapy according to their diagnosis subtype that may lead to divergent effects on their clinical outcomes in the context of AMI. The majority of studies have either investigated the overall effect of all-cause SMI $\underline{11} \underline{14} \underline{15}$ or analyzed individual mental health subtypes without a comparison of the relative risks of clinical outcomes according to SMI subtype.10 Finally, while some studies have looked at gender differences in reperfusion therapy $\underline{11} \underline{12} \underline{16}$, there have been no reports on differences in clinical outcomes of SMI patients after AMI according to gender.

The current study utilized a national cohort drawn from the National Inpatient Sample over an eleven-year period (2004-2014) to define the prevalence of patients with SMI presenting with AMI and examine their clinical characteristics, in-hospital outcomes and processes of care, stratified by subtype of SMI and further stratified by gender.

\section{Methods}

\section{Data Source}


The National Inpatient Sample (NIS), is the largest publicly available all-payer database of hospitalized patients in the United States and is sponsored by the Agency for Healthcare Research and Quality as a part of the Healthcare Cost and Utilization Project. 17 Further information about the design of NIS and its previous validation is available in the supplementary materials (Appendix A) .

\section{Study Design and Population}

A retrospective observational analysis of all AMI hospitalizations between 2004 and 2014 from the NIS database was performed. Diagnoses and procedure variables were extracted using the relevant International Classification of Diseases, 9th Revision, Clinical Modification (ICD-9 CM) codes. The cohort was stratified into 5 groups; 'No SMI', Schizophrenia, Bipolar Disorder, 'Other non-organic psychoses' (ONOP) and 'Major Depression'. Further information on the methods used to identify patient characteristics and procedural data and exclude missing cases is available in the supplementary material (Appendix B and Table S1)

\section{Outcomes}

The primary outcome measures were to define the prevalence of SMI in AMI and examine the clinical characteristics, comorbidity burden and in-hospital rates of major acute cardiovascular and cerebrovascular events (MACCE) (composite of mortality, acute stroke/transient ischemic attack (TIA) and cardiac complications) and all-cause bleeding. Cardiac complications included any pericardiocentesis, cardiac tamponade, hemopericardium and coronary dissection during hospitalization, while. bleeding was defined as any postprocedural hemorrhage or hematoma, or bleeding requiring transfusion. 
The secondary outcome was the evaluation of the receipt of coronary angiography (CA) and revascularization (percutaneous coronary intervention (PCI) and coronary artery bypass grafting $(\mathrm{CABG}))$.

\section{Statistical Analysis}

For detailed statistical analysis methods and a list of the covariates adjusted for please refer to the supplementary material (Appendix C)

\section{Results}

A total of $6,738,757$ patients were included in the final analysis (Figure S1). Patients with a diagnosis of SMI comprised $6.5 \%(n=439,544)$ of the study cohort. The patient characteristics of the study population are presented in the supplements (Table S2) according to the presence or absence of SMI, and SMI subtype; Schizophrenia $(0.4 \%, \mathrm{n}=23,582)$, Bipolar Disorder $(0.3 \%, \mathrm{n}=41,362)$, ONOP $(0.6 \%, \mathrm{n}=22,359)$ and Major Depression $(5.3 \%$, $\mathrm{n}=352,241$ ), the latter being the most common subtype. The prevalence of SMI amongst the ACS population doubled over the study period (from $4.5 \%$ in 2004 to $9.5 \%$ in 2014), primarily due to an increase in Major Depression and Bipolar Disorder diagnoses (Figure 1), while the prevalence of ONOP and Schizophrenia disorders has not changed.

Several key differences in patient demographics are seen across the study groups. (Table S1, Appendix D)

\section{Utilization of invasive management}

There was a notable increase in the rates of both coronary angiography and PCI in all the groups over the study period (Figure S2). However, in comparison to patients without SMI, the rates of coronary angiography and PCI were persistently lower in all SMI subtypes except those with Bipolar Disorder, whom overall were more likely to be offered coronary 
angiography $(67.1 \%$ vs. $64.4 \%, \mathrm{p}<0.001)$ (Figure 2, Table S4). In multivariate analysis, all SMI subtypes were associated with reduced odds of coronary angiography and PCI, with Schizophrenia patients being the least likely to undergo either of the procedures (Figure 6, Table S6).

The rates of coronary angiography and PCI were also lower amongst women across all study groups, including patients without an SMI diagnosis (Figure 3), a finding that persisted in multivariate analysis (Table S7). However, the odds of receipt of coronary angiography between genders were not significant in the Schizophrenia and Major Depression groups.

\section{In-hospital MACCE, Mortality and Stroke}

Patients with ONOP and Schizophrenia experienced the highest rates of in-hospital MACCE (Figure 4), primarily driven by higher rates of mortality (6.4\% and $7.5 \%)$ and stroke (4.0\% and $2.2 \%$ ) compared to other SMI subtypes and patients without SMI $(\mathrm{p}<0.001$ for both). The lowest rates of MACCE, mortality and stroke were observed in patients with Bipolar Disorder (Table 1).

In multivariate analysis, patients with ONOP and Major Depression were associated with significantly reduced odds of $\operatorname{MACCE}(0.86[0.82,0.90]$ and $0.86[0.85,0.88]$, respectively) (Figure 6, Table S6). However, both groups demonstrated a polar effect on Stroke, where patients with ONOP were at 53\% increased odds of stroke and those with Major Depression associated with $16 \%$ reduced odds of stroke. There was no difference in either outcome (MACCE and stroke) in patients with Schizophrenia and Bipolar Disorder. Schizophrenia was the only subtype associated with increased odds of mortality (1.10 [1.04, 1.16]), while all other SMI subtypes were associated with a significantly reduced odds of mortality. 
In subgroup gender analysis, the rates of in-hospital MACCE, mortality and Stroke were consistently higher in women compared to men, except the rates of stroke in ONOP that were higher in men. (Figure 5, Table S4) In multivariable analysis, the odds of MACCE were increased in women without SMI and those with Bipolar Disorder and Major Depression, primarily driven by increased odds of acute stroke in these groups. (Table S7) In contrast to the other groups, women with ONOP were associated with reduced odds of MACCE and stroke compared to men (Table S7). Furthermore, women without SMI and those with ONOP and Major Depression were at reduced odds of mortality compared to men, while there was no difference in mortality between genders in the Schizophrenia and Bipolar Disorder groups.

A temporal analysis of outcomes demonstrates an overall decline in the rates of MACCE, mortality and acute stroke/TIA in all the study groups except the ONOP and Bipolar disorder groups whose rates of MACCE and acute stroke/TIA have either increased or remained the same (Table S5). However, the pattern of differences in rates of MACCE, morality and stroke between groups remained the same throughout the study period.

\section{In-hospital Bleeding}

In comparison to patients without SMI, only the ONOP group experienced higher rates of bleeding, while all other SMI subtypes experienced lower rates of bleeding (Figure 4). Subgroup gender analysis demonstrated higher rates of bleeding amongst women in patients without SMI and those with Schizophrenia, and lower rates of bleeding amongst women in patients with Bipolar Disorder and ONOP. (Figure 5) There was no difference in crude rates of bleeding between both genders in patients with Major Depression.

In multivariate analysis, only patients with ONOP were associated with an increased odds of bleeding $(1.11[1.04,1.17], \mathrm{p}=0.001)$ while all other SMI subtypes were at a significantly reduced odds of bleeding (Schizophrenia: 0.70 [0.65, 0.75]; Bipolar Disorder: 
0.85 [0.81, 0.90]; Major Depression: 0.97 [0.95,0.98], $\mathrm{p}<0.001$ for all outcomes) (Figure 6, Table S6).

A multivariate analysis according to gender revealed increased odds of bleeding in women without SMI as well as those with Schizophrenia and Major Depression (Supplementary Material - Table S7). In contrast, no difference in bleeding was found between genders in patients with ONOP and women with Bipolar disorder were at reduced odds of bleeding.

The rate of all-cause bleeding increased in all groups over the study period but remained higher in SMI groups compared to patients without SMI, with the exception of ONOP group whose rates of bleeding were higher than patients without SMI. (Table S5)

\section{Sensitivity Analysis}

A sensitivity analysis according to AMI type (STEMI vs. NSTEMI) was performed to exclude examine differences in outcomes between both AMI presentations. Overall, the findings in the STEMI and NSTEMI groups were generally similar to those found in the total cohort. (Table S6) However, certain differences were observed within AMI subgroups. In the NSTEMI subgroup, patients with Bipolar Disorder were associated with reduced odds of MACCE compared to those without SMI while there was no difference in MACCE and allcause bleeding between patients without SMI and those with ONOP. In the STEMI subgroup, there was no difference in mortality and all-cause bleeding between patients with Bipolar Disorder group and those without SMI as was the case in all-cause bleeding between patients with Major Depression and those without SMI. 


\section{Discussion}

Our findings in the present study, drawn from a national cohort of acute coronary syndrome hospitalizations in the United States, show a significant rise in the prevalence of SMI patients presenting with AMI over the study period. We observe that patients with SMI represent a multi-morbid cohort that is higher in cardiovascular risk with a more significant burden of comorbidities than those without SMI. Our results demonstrate significant disparities in the clinical outcomes and utilization of invasive strategies amongst patients with severe mental illnesses, including Bipolar Disorder, ONOP, Schizophrenia and Major Depression. Furthermore, we report lower rates of invasive management (coronary angiography and PCI) in patients with SMI, who experience higher crude rates of adverse outcomes, including MACCE, mortality, bleeding and stroke, compared to those without a SMI diagnosis. However, after adjustment for confounders, patients with Schizophrenia were associated with increased odds of in-hospital mortality amongst all SMI subtypes, whilst patients with ONOP were the only SMI group at increased odds of stroke and bleeding complications following AMI. Finally, our analysis highlights a gender gap in clinical outcomes and invasive management strategy, with women being less likely to be offered early invasive strategies (coronary angiography and PCI) despite their higher risk of mortality.

Patients with SMI are a heterogenous group with a multitude of pathological and socioeconomic risk factors for cardiovascular disease, depending on the subtype of SMI, and been shown to have a higher burden of risk factors such as diabetes, smoking, obesity and chronic kidney disease. $\underline{18-23}$ Furthermore, their use of psychotropic medications such as antipsychotics and selective serotonin reuptake inhibitors (SSRIs) places them at a higher risk of adverse outcomes including stroke, cardiovascular mortality and bleeding. $\frac{24-27}{\underline{2}}$ Antipsychotics and antidepressants such as SSRI's (selective serotonin reuptake inhibitors) 
have been shown to increase the risk of metabolic syndrome due to their interaction with target 5-HT neuroreceptors that modulate weight control and glucose metabolism, which increases the risk of myocardial infarction.28-30 Furthermore, the use of antipsychotics (typical and atypical) has been associated with an increased risk of cerebrovascular events, especially in elderly patients, although the underlying mechanism remains unknown, $\underline{31}$ ' $\underline{32}$, while SSRI's are thought to increase the uptake of serotonin into platelets leading to increased bleeding tendency. $\underline{33}$ In this national analysis of all hospitalizations with AMI in the US, we show that only more complex SMI subtypes such Schizophrenia were at increased odds of in-hospital mortality. A previous national analysis of the SWEDEHEART registry also demonstrated higher mortality in patients with Schizophrenia both at 30-day (OR 2.58 [1.88-3.54)) and one-year (OR: 2.97 [2.39-3.69]) follow up in patients admitted following AMI.12 However, in contrast to our findings, patients with Bipolar Disorder $(n=442)$ were also shown to be at greater odds of mortality in their analysis albeit only significant at 1-year follow-up (OR 1.62 [1.20-2.20]). In contrast, an analysis of approximately 800 veterans with SMI showed lower crude rates of 30 day mortality (4.6\% vs. 6.2\%) and higher 1-year mortality (15.3\% vs. $13.2 \%)$ in SMI patients compared to those without any mental illness. 14 While their findings did not reach statistical significance, the marked difference in results between studies highlights the limitations of analyzing small and combined cohorts of all SMI. More importantly, not all the SMI subtypes are associated with increased mortality hazard in our analysis, with certain SMI subtypes such as Bipolar Disorder and Major Depression being at lower odds of in-hospital mortality compared to those without SMI.

To the best of our knowledge, no previous study has examined stroke and bleeding outcomes in SMI patients after AMI. Our findings show that only patients with ONOP were at increased odds of bleeding and stroke, while all other SMI subtypes (Bipolar Disorder, Schizophrenia and Major Depression) were at lower odds of both outcomes compared to no 
SMI. A plausible explanation could be the greater use of psychotropic medications in patients with psychosis that is known to be associated with increased odds of adverse cardiovascular events, especially in elderly patients, although the underlying mechanism remains unknown. 31' 32 Similarly, the use of selective serotonin reuptake inhibitors (SSRI's) is known to promote the uptake of serotonin into platelets leading to an increase in bleeding tendency. $\underline{33}$ The lower frequency of stroke and bleeding in SMI subtypes other than ONOP could be explained by the low rates of invasive coronary management in these patients. Although we have adjusted for the differences in patient demographics and receipt of revascularization, there could be residual unmeasured confoundment that accounts for these findings.

Our gender subgroup analysis highlights persistent differences in invasive management strategies and in clinical outcomes of both SMI and non-SMI patients. We found women in all study group to be associated with lower or insignificant odds of mortality compared to men, but also at increased odds of acute stoke/TIA, except in those with ONOP where the was no difference between genders. Women with Schizophrenia were also associated with the highest odds of bleeding complications compared to men. However, women of all subgroups were much less likely to undergo invasive management compared to men, especially women with Bipolar Disorder who were $15 \%$ and $34 \%$ less likely to receive coronary angiography and PCI, respectively. Despite the lack of evidence on gender outcomes in this population, a possible explanation for our findings is the higher prevalence of comorbidities predisposing to bleeding and stroke amongst women as seen in our patients' demographics. However, the disparities in clinical outcomes according to gender highlights the need for prospective studies to explore the underlying pathophysiological mechanisms that may lead to these differences in patients with SMI.

The present study is the first to examine the prevalence of SMI in the AMI population and shows a rise in the prevalence of SMI over an eleven-year horizon, primarily due 
increasing rates of Major Depression and Bipolar disorder diagnoses. Although these findings are in keeping with previous reports of trends in the general population, $\underline{34-36}$ it is possible that that these diagnoses have been coded more frequently in later years due to physicians' awareness of their implications in the context of AMI hospitalizations.

The lower rates of invasive management (coronary angiography and PCI) in patients with SMI may be due to several reasons. It is possible that patients suffering from SMI lack the ability to engage in the discussions required for informed consent for such procedures. Furthermore, their lack of recognition and diagnostic overshadowing in emergency services leads to delayed presentations of their acute myocardial infarction. Patients undergoing PCI are committed to a minimum duration of dual antiplatelet therapy as well as other secondary prevention pharmacotherapy, regular follow up and access to cardiac rehabilitation to optimize their response to pharmacotherapy and success of their coronary intervention $\underline{37}, \underline{38}$ However, patients with SMI are previously reported to have poor compliance with diet advice, cardiac medications and rehabilitation, which often leads to physicians' reluctance to offer them coronary intervention. $\underline{4} \underline{10} \underline{15}$ Consequently, patients with SMI who do not undergo in-hospital invasive management are at an even greater risk of adverse clinical outcomes after AMI, including higher rates of mortality and future re-infarction. $\underline{37-39}$ However, only few studies have compared these management strategies across different SMI subtypes, while the majority have examined the association of all-cause SMI with odds of reperfusion therapy. $\underline{10} \underline{11} \underline{15}$ For example, Campi et al. reported reduced odds of reperfusion therapy (PCI, thrombolysis or CABG; OR $0.70[0.60,0.80]$ ) in patients with charted mental illnesses (composite of Schizophrenia, Bipolar, and Depressive disorders; $\mathrm{n}=1036$ ) presenting with AMI across the state of Florida. 11 While these findings were significant, they did not inform of SMI subgroups with the least odds of receiving invasive coronary management. We show that patients with SMI of all subtypes were consistently less 
likely to be offered an invasive management strategy (coronary angiography and PCI) over the study decade $\stackrel{40-42}{2}$ The lowest odds of invasive management (coronary angiography and PCI) were in patients with Schizophrenia, although they were at the greatest risk of mortality in our study. An individualized patient-based approach is required to assess these complex patients to improve utilization of guideline-recommended therapy in this high-risk group.

\section{Limitations}

The are several limitations to the present study. First, the NIS is an administrative dataset that is susceptible to coding. Although the identification of ACS and SMI diagnoses as well as other comorbidities was based on the use of administrative codes, ICD-9 codes

have been previously validated for the purposes of cardiovascular research. ${ }^{43}, \underline{44}$ Second, the ischemic benefits of an invasive strategy over a conservative management of AMI have been primarily shown in studies reporting at least 30 -day follow up. $\underline{39}, \underline{45}, \underline{46}$ Therefore, it is possible that SMI patients experience worse clinical outcomes in the long term than those reported in the present study that only captures in-hospital outcomes. Third, since the NIS dataset does not capture pharmacotherapy, we were unable to determine differences in the use of antithrombotic therapy between the study groups. Finally, we have not been able to assess the severity of the mental health illness or the overlap of mental health diagnoses, which remain as potential residual confounders. Nevertheless, we believe that our findings provide insight into the 'real world' in-hospital clinical outcomes of a large and unselected ACS cohort with severe mental illness.

\section{Conclusion}

Our analysis a national cohort of acute coronary syndrome hospitalizations shows a disparity of clinical outcomes amongst patients with severe mental illness and identifies 
patients with Schizophrenia and 'other non-organic psychoses' as those with the poorest outcomes, namely higher in-hospital mortality and in-hospital stroke, respectively. Furthermore, we conclude that patients with SMI are less likely to be offered an invasive management strategy, with women of all SMI subtypes and patients with Schizophrenia being the most disadvantaged groups.

\section{Conflicts}

DS is expert advisor to the NICE centre for guidelines and a member of the current NICE guideline development group for Rehabilitation in adults with complex psychosis and related severe mental health conditions; Board member of the National Collaborating Centre for Mental Health (NCCMH); views are personal and not those of NICE or NCCMH.

\section{Funding}

MOM receives PhD studentship funding from Medtronic Ltd.

\section{References}

1. Crump C, Sundquist K, Winkleby MA, Sundquist J. Comorbidities and mortality in bipolar disorder: a Swedish national cohort study. JAMA psychiatry. 2013;70:931939.

2. Laursen TM, Wahlbeck K, Hallgren J, et al. Life expectancy and death by diseases of the circulatory system in patients with bipolar disorder or schizophrenia in the Nordic countries. PLoS ONE. 2013;8:e67133.

3. Laursen TM, Nordentoft M. Heart disease treatment and mortality in schizophrenia and bipolar disorder - changes in the Danish population between 1994 and 2006. J. Psychiatr. Res. 2011;45:29-35.

4. Osborn DJ, Levy G, Nazareth I, Petersen I, Islam A, King MB. Relative risk of cardiovascular and cancer mortality in people with severe mental illness from the united kingdom general practice research database. Arch. Gen. Psychiatry. 2007;64:242-249.

5. Kessler RC, Aguilar-Gaxiola S, Alonso J, et al. The global burden of mental 
disorders: an update from the WHO World Mental Health (WMH) surveys. Epidemiol. Psichiatr. Soc. 2009;18:23-33.

6. Administration. SAaMHS. Key substance use and mental health indicators in the United States: Results from the 2016 National Survey on Drug Use and Health Vol 2019: Center for Behavioral Health Statistics and Quality, Substance Abuse and Mental Health Services Administration. ; 2017.

7. Wu S-I, Chen S-C, Liu S-I, et al. Relative Risk of Acute Myocardial Infarction in People with Schizophrenia and Bipolar Disorder: A Population-Based Cohort Study. PLoS ONE. 2015;10:e0134763.

8. Kanuch SW, Cassidy KA, Dawson NV, Athey M, Fuentes-Casiano E, Sajatovic M. Recruiting and Retaining Individuals with Serious Mental Illness and Diabetes in Clinical Research: Lessons Learned from a Randomized, Controlled Trial. Journal of health disparities research and practice. 2016;9:115-126.

9. Schulman-Marcus J, Goyal P, Swaminathan RV, et al. Comparison of Trends in Incidence, Revascularization, and In-Hospital Mortality in ST-Elevation Myocardial Infarction in Patients With Versus Without Severe Mental Illness. Am. J. Cardiol. 2016;117:1405-1410.

10. Druss BG, Bradford DW, Rosenheck RA, Radford MJ, Krumholz HM. Mental disorders and use of cardiovascular procedures after myocardial infarction. JAMA. 2000;283:506-511.

11. Campi TR, Jr., George S, Villacís D, Ward-Peterson M, Barengo NC, Zevallos JC. Effect of charted mental illness on reperfusion therapy in hospitalized patients with an acute myocardial infarction in Florida. Medicine (Baltimore). 2017;96.

12. Bodén R, Molin E, Jernberg T, Kieler H, Lindahl B, Sundström J. Higher mortality after myocardial infarction in patients with severe mental illness: a nationwide cohort study. J. Intern. Med. 2014;277:727-736.

13. Attar R, Berg Johansen M, Valentin JB, Aagaard J, Jensen SE. Treatment following myocardial infarction in patients with schizophrenia. PLoS ONE. 2017;12:e0189289.

14. Petersen LA, Normand S-LT, Druss BG, Rosenheck RA. Process of care and outcome after acute myocardial infarction for patients with mental illness in the VA health care system: are there disparities? Health Serv. Res. 2003;38:41-63.

15. Li Y, Glance LG, Lyness JM, Cram P, Cai X, Mukamel DB. Mental Illness, Access to Hospitals with Invasive Cardiac Services, and Receipt of Cardiac Procedures by Medicare Acute Myocardial Infarction Patients. Health Serv. Res. 2012;48:10761095.

16. Parashar S, Rumsfeld JS, Reid KJ, et al. Impact of depression on sex differences in outcome after myocardial infarction. Circ. Cardiovasc. Qual. Outcomes. 2009;2:3340.

17. Agency for Healthcare Research and Quality R, MD. HCUP NIS Database Documentation. Healthcare Cost and Utilization Project (HCUP).February 2018.

18. Pan A, Sun Q, Okereke OI, Rexrode KM, Hu FB. Depression and risk of stroke morbidity and mortality: A meta-analysis and systematic review. JAMA. 2011;306:1241-1249.

19. Birkenaes AB, Opjordsmoen $\mathrm{S}$, Brunborg $\mathrm{C}$, et al. The level of cardiovascular risk factors in bipolar disorder equals that of schizophrenia: a comparative study. J. Clin. Psychiatry. 2007;68:917-923.

20. Goff DC, Sullivan LM, McEvoy JP, et al. A comparison of ten-year cardiac risk estimates in schizophrenia patients from the CATIE study and matched controls. Schizophr. Res. 2005;80:45-53.

21. De Hert M, Correll CU, Solmi M, et al. Prevalence, incidence and mortality from 
cardiovascular disease in patients with pooled and specific severe mental illness: a large-scale meta-analysis of 3,211,768 patients and 113,383,368 controls. World Psychiatry. 2017;16:163-180.

22. Bahorik AL, Satre DD, Kline-Simon AH, Weisner CM, Campbell CI. Serious mental illness and medical comorbidities: Findings from an integrated health care system. $J$. Psychosom. Res. 2017;100:35-45.

23. Zolezzi M, Abdulrhim S, Isleem N, Zahrah F, Eltorki Y. Medical comorbidities in patients with serious mental illness: a retrospective study of mental health patients attending an outpatient clinic in Qatar. Neuropsychiatric disease and treatment. 2017;13:2411-2418.

24. Tiihonen J, Mittendorfer-Rutz E, Torniainen M, Alexanderson K, Tanskanen A. Mortality and Cumulative Exposure to Antipsychotics, Antidepressants, and Benzodiazepines in Patients With Schizophrenia: An Observational Follow-Up Study. Am. J. Psychiatry. 2016;173:600-606.

25. Straus SM, Bleumink GS, Dieleman JP, et al. Antipsychotics and the risk of sudden cardiac death. Arch. Intern. Med. 2004;164:1293-1297.

26. Douglas IJ, Smeeth L. Exposure to antipsychotics and risk of stroke: self controlled case series study. BMJ. 2008;337.

27. Jiang H-Y, Chen H-Z, Hu X-J, et al. Use of Selective Serotonin Reuptake Inhibitors and Risk of Upper Gastrointestinal Bleeding: A Systematic Review and Metaanalysis. Clin. Gastroenterol. Hepatol. 2015;13:42-50.e43.

28. Casey DE, Zorn SH. The pharmacology of weight gain with antipsychotics. J. Clin. Psychiatry. 2001;62 Suppl 7:4-10.

29. Citrome L, Jaffe A, Levine J, Allingham B, Robinson J. Relationship between antipsychotic medication treatment and new cases of diabetes among psychiatric inpatients. Psychiatr. Serv. 2004;55:1006-1013.

30. Serretti A, Mandelli L. Antidepressants and body weight: a comprehensive review and meta-analysis. J. Clin. Psychiatry. 2010;71:1259-1272.

31. Gill SS, Rochon PA, Herrmann N, et al. Atypical antipsychotic drugs and risk of ischaemic stroke: population based retrospective cohort study. BMJ. 2005;330:445.

32. Wang PS, Schneeweiss S, Avorn J, et al. Risk of Death in Elderly Users of Conventional vs. Atypical Antipsychotic Medications. N. Engl. J. Med. 2005;353:2335-2341.

33. Hallbäck I, Hägg S, Eriksson AC, Whiss PA. In vitro effects of serotonin and noradrenaline reuptake inhibitors on human platelet adhesion and coagulation. Pharmacol. Rep. 2012;64:979-983.

34. Sara GE, Malhi GS. Trends in diagnosis of bipolar disorder: Have the boundaries changed? Aust. N. Z. J. Psychiatry. 2015;49:1021-1028.

35. Weinberger AH, Gbedemah M, Martinez AM, Nash D, Galea S, Goodwin RD. Trends in depression prevalence in the USA from 2005 to 2015: widening disparities in vulnerable groups. Psychol. Med. 2018;48:1308-1315.

36. Jeuring HW, Comijs HC, Deeg DJH, Stek ML, Huisman M, Beekman ATF. Secular trends in the prevalence of major and subthreshold depression among 55-64-year olds over 20 years. Psychol. Med. 2018;48:1824-1834.

37. Roffi M, Patrono C, Collet JP, et al. 2015 ESC Guidelines for the management of acute coronary syndromes in patients presenting without persistent ST-segment elevation: Task Force for the Management of Acute Coronary Syndromes in Patients Presenting without Persistent ST-Segment Elevation of the European Society of Cardiology (ESC). Eur. Heart J. 2016;37:267-315.

38. Amsterdam EA, Wenger NK, Brindis RG, et al. 2014 AHA/ACC Guideline for the 
Management of Patients with Non-ST-Elevation Acute Coronary Syndromes: a report of the American College of Cardiology/American Heart Association Task Force on Practice Guidelines. J. Am. Coll. Cardiol. 2014;64:e139-e228.

39. Milosevic A, Vasiljevic-Pokrajcic Z, Milasinovic D, et al. Immediate Versus Delayed Invasive Intervention for Non-STEMI Patients: The RIDDLE-NSTEMI Study. JACC Cardiovasc. Interv. 2016;9:541-549.

40. Ibanez B, James S, Agewall S, et al. 2017 ESC Guidelines for the management of acute myocardial infarction in patients presenting with ST-segment elevation: The Task Force for the management of acute myocardial infarction in patients presenting with ST-segment elevation of the European Society of Cardiology (ESC). Eur. Heart J. 2018;39:119-177.

41. Jneid H, Addison D, Bhatt DL, et al. 2017 AHA/ACC Clinical Performance and Quality Measures for Adults With ST-Elevation and Non-ST-Elevation Myocardial Infarction: A Report of the American College of Cardiology/American Heart Association Task Force on Performance Measures. J. Am. Coll. Cardiol. 2017;70:2048-2090.

42. Ibanez B, James S, Agewall S, et al. 2017 ESC Guidelines for the management of acute myocardial infarction in patients presenting with ST-segment elevationThe Task Force for the management of acute myocardial infarction in patients presenting with ST-segment elevation of the European Society of Cardiology (ESC). European Heart Journal. 2018;39:119-177.

43. Birman-Deych E, Waterman AD, Yan Y, Nilasena DS, Radford MJ, Gage BF. Accuracy of ICD-9-CM Codes for Identifying Cardiovascular and Stroke Risk Factors. Med Care. 2005;43.

44. DeShazo JP, Hoffman MA. A comparison of a multistate inpatient EHR database to the HCUP Nationwide Inpatient Sample. BMC Health Serv. Res. 2015;15:384.

45. Borgia F, Goodman SG, Halvorsen S, et al. Early routine percutaneous coronary intervention after fibrinolysis vs. standard therapy in ST-segment elevation myocardial infarction: a meta-analysis. Eur. Heart J. 2010;31:2156-2169.

46. Brodie BR, Stuckey TD, Wall TC, et al. Importance of time to reperfusion for 30-day and late survival and recovery of left ventricular function after primary angioplasty for acute myocardial infarction. J. Am. Coll. Cardiol. 1998;32:1312-1319. 
Table 1. In-hospital clinical outcomes and Quality Indicators according to subtype of SMI

\begin{tabular}{|c|c|c|c|c|c|c|c|}
\hline & No SMI & Schizophrenia & ONOP & Bipolar disorder & $\begin{array}{c}\text { Major } \\
\text { depression }\end{array}$ & Total & $\begin{array}{c}\mathrm{p}- \\
\text { value }\end{array}$ \\
\hline MACCE, \% & 8.0 & 9.6 & 10.6 & 5.6 & 6.7 & 7.9 & $<0.001$ \\
\hline $\begin{array}{l}\text { Cardiac } \\
\text { complications, \% }\end{array}$ & 0.7 & 0.4 & 0.6 & 0.8 & 0.6 & 0.7 & $<0.001$ \\
\hline Acute stroke/TIA, \% & 1.7 & 2.2 & 4.0 & 1.5 & 1.8 & 1.7 & $<0.001$ \\
\hline Mortality, \% & 6.0 & 7.5 & 6.4 & 3.4 & 4.6 & 5.9 & $<0.001$ \\
\hline $\begin{array}{l}\text { All-cause bleeding, } \\
\%\end{array}$ & 5.3 & 3.8 & 6.3 & 4.4 & 5.1 & 5.2 & $<0.001$ \\
\hline $\begin{array}{l}\text { Use of assist device } \\
\text { or IABP, \% }\end{array}$ & 5.0 & 4.0 & 4.9 & 3.9 & 3.3 & 4.9 & $<0.001$ \\
\hline Shock, \% & 5.0 & 6.1 & 5.4 & 4.0 & 3.6 & 4.9 & $<0.001$ \\
\hline $\begin{array}{l}\text { Receipt of coronary } \\
\text { angiography, \% }\end{array}$ & 64.4 & 46.8 & 42.9 & 67.1 & 59.5 & 64.0 & $<0.001$ \\
\hline Receipt of $\mathrm{PCl}, \%$ & 42.9 & 27.2 & 23.0 & 42.4 & 36.9 & 42.5 & $<0.001$ \\
\hline Receipt of CABG, \% & 8.5 & 5.4 & 10.9 & 7.0 & 6.9 & 8.4 & $<0.001$ \\
\hline $\begin{array}{l}\text { Discharge against } \\
\text { medical advice (\%) }\end{array}$ & 0.8 & 2.5 & 1.5 & 2.7 & 0.8 & 0.8 & $<0.001$ \\
\hline
\end{tabular}

CABG: coronary artery bypass grafting; MACCE: major adverse cardiovascular and cerebrovascular events (composite of mortality, acute stroke/transient and cardiac complications); ONOP: Other Non-Organic Psychoses; PCI: percutaneous coronary angiography; TIA: transient ischemic attack 


\section{Figure Legends}

Figure 1. Prevalence of SMI from 2004 to 2014 according to subtype

Legend: ONOP: Other non-organic psychoses

Figure 2. Receipt of coronary angiography (CA) and percutaneous coronary intervention (PCI)

Legend: ONOP: Other non-organic psychoses; SMI: Severe mental illness

Figure 3. Receipt of coronary angiography (CA) and PCI according to SMI subtype and gender

Legend: ONOP: Other non-organic psychoses; SMI: Severe mental illness

Figure 4. In-hospital adverse events according to SMI subtype

Legend: ONOP: Other non-organic psychoses; SMI: Severe mental illness

Figure 5. In-hospital adverse events according to gender

Legend: ONOP: Other non-organic psychoses; SMI: Severe mental illness

Figure 6. Odds ratio (95\% CI) of adverse outcomes according to SMI subtype Legend: *Reference group is No-SMI; $\S$ non-significant $(\mathrm{p} \geq 0.05) ; \dagger \mathrm{p}<0.001$; CI: confidence interval; ONOP: Other non-organic psychoses 


\begin{tabular}{|c|c|c|c|c|c|c|c|c|c|c|c|}
\hline \multicolumn{12}{|l|}{$12 \%$} \\
\hline \multirow{2}{*}{\multicolumn{12}{|c|}{$10 \%$}} \\
\hline & & & & & & & & & & & \\
\hline \multicolumn{12}{|l|}{$6 \%$} \\
\hline \multicolumn{12}{|l|}{$2 \%$} \\
\hline \multirow{2}{*}{$0 \%$} & & & & & & & & & & & \\
\hline & 2004 & 2005 & 2006 & 2007 & 2008 & 2009 & 2010 & 2011 & 2012 & 2013 & 2014 \\
\hline Major Depression & $3.4 \%$ & $3.5 \%$ & $3.8 \%$ & $4.1 \%$ & $4.6 \%$ & $5.0 \%$ & $5.2 \%$ & $5.8 \%$ & $7.0 \%$ & $7.7 \%$ & $8.1 \%$ \\
\hline$\square$ Bipolar & $0.4 \%$ & $0.3 \%$ & $0.4 \%$ & $0.5 \%$ & $0.6 \%$ & $0.6 \%$ & $0.7 \%$ & $0.8 \%$ & $0.8 \%$ & $0.8 \%$ & $0.9 \%$ \\
\hline EOther Non-Organic Psychoses & $0.3 \%$ & $0.3 \%$ & $0.3 \%$ & $0.3 \%$ & $0.3 \%$ & $0.3 \%$ & $0.4 \%$ & $0.4 \%$ & $0.4 \%$ & $0.4 \%$ & $0.3 \%$ \\
\hline घSchizophrenia & $0.4 \%$ & $0.3 \%$ & $0.4 \%$ & $0.3 \%$ & $0.3 \%$ & $0.3 \%$ & $0.4 \%$ & $0.4 \%$ & $0.3 \%$ & $0.4 \%$ & $0.3 \%$ \\
\hline
\end{tabular}


Click here to download high resolution image

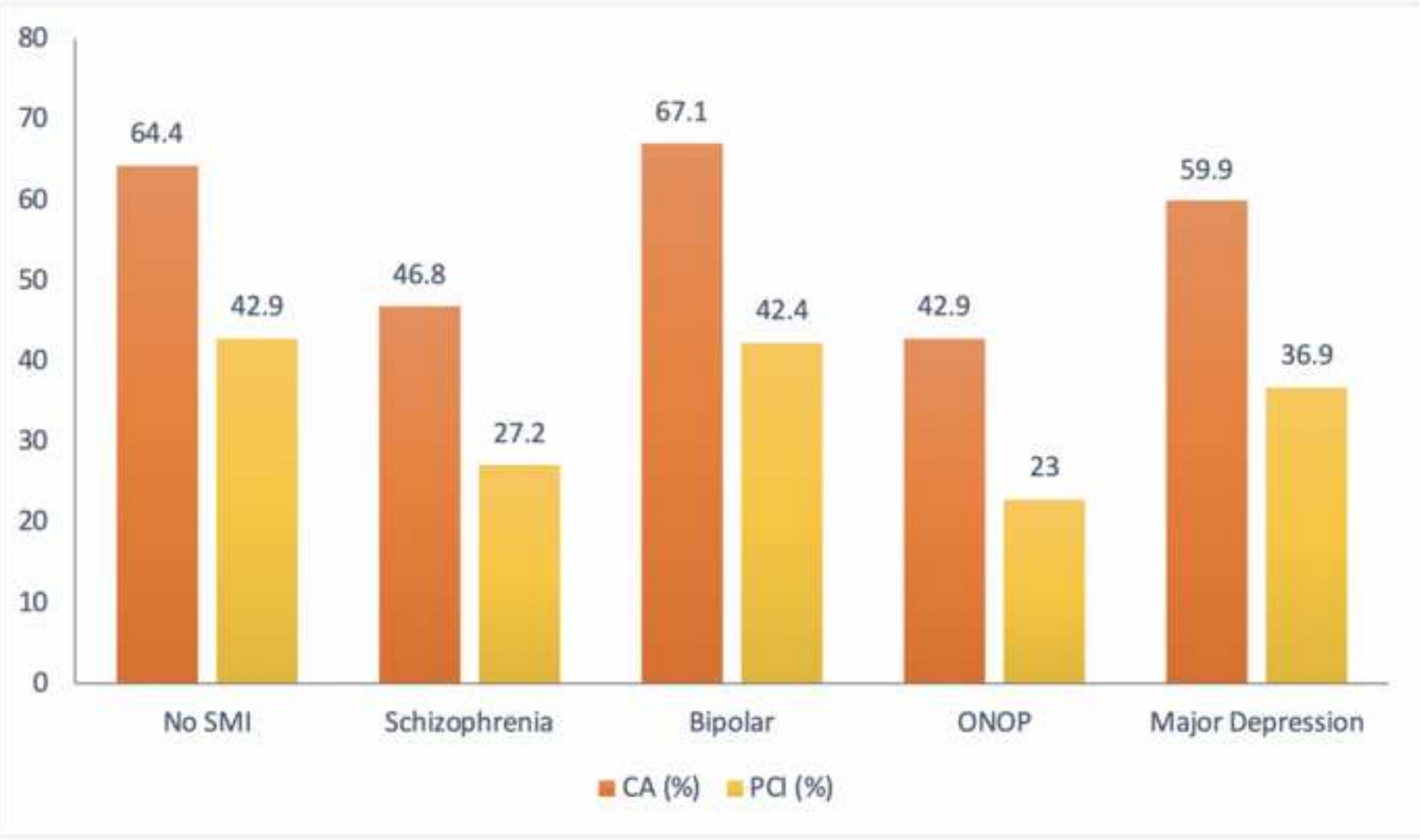


Click here to download high resolution image
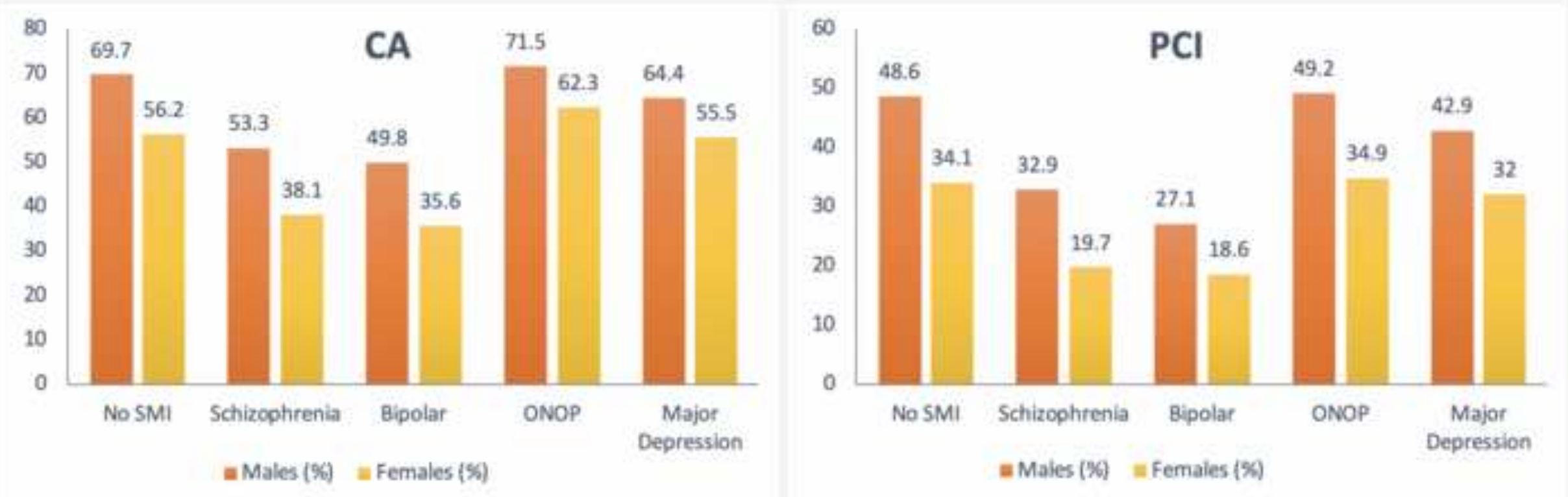


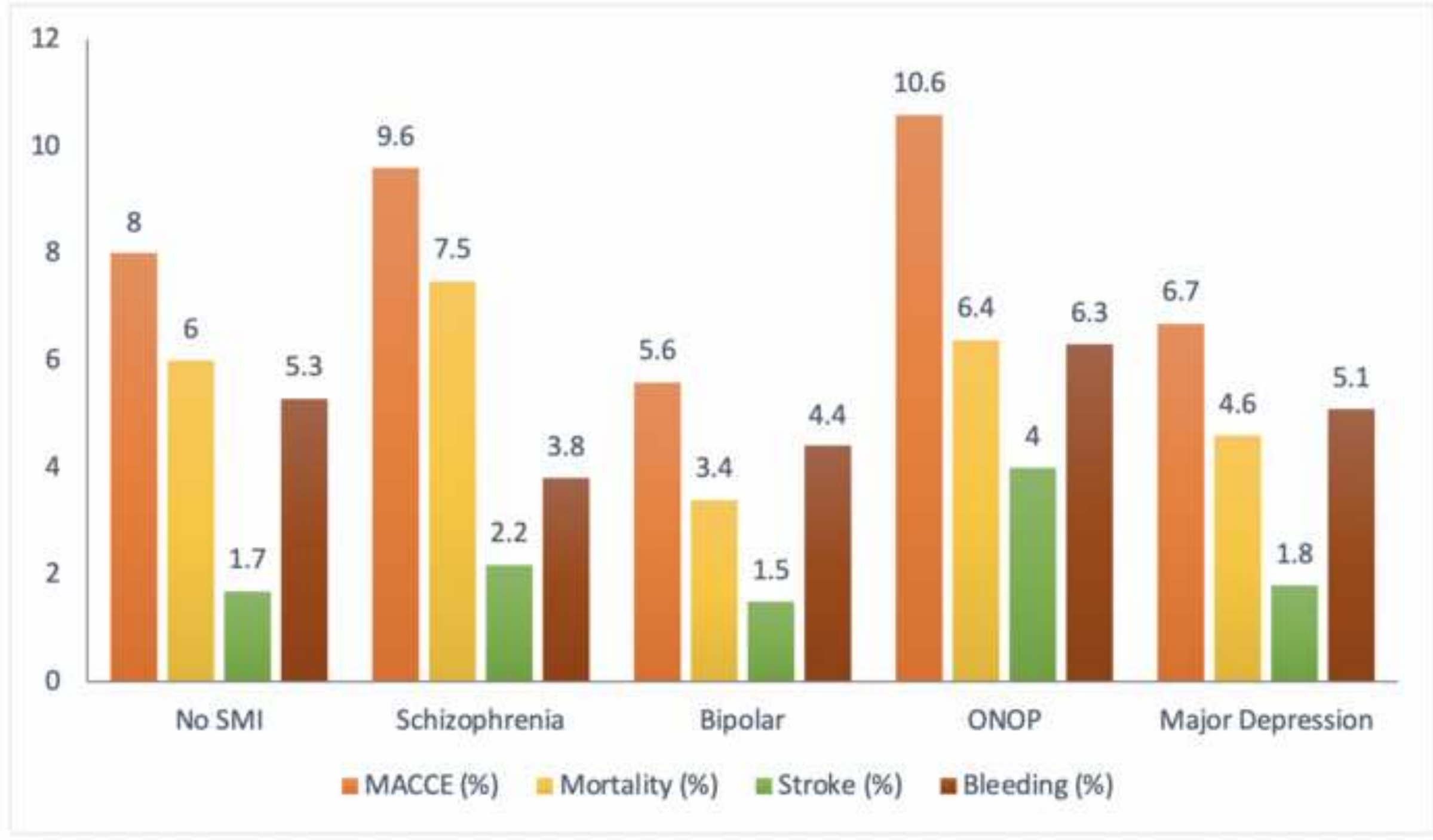


Click here to download high resolution image

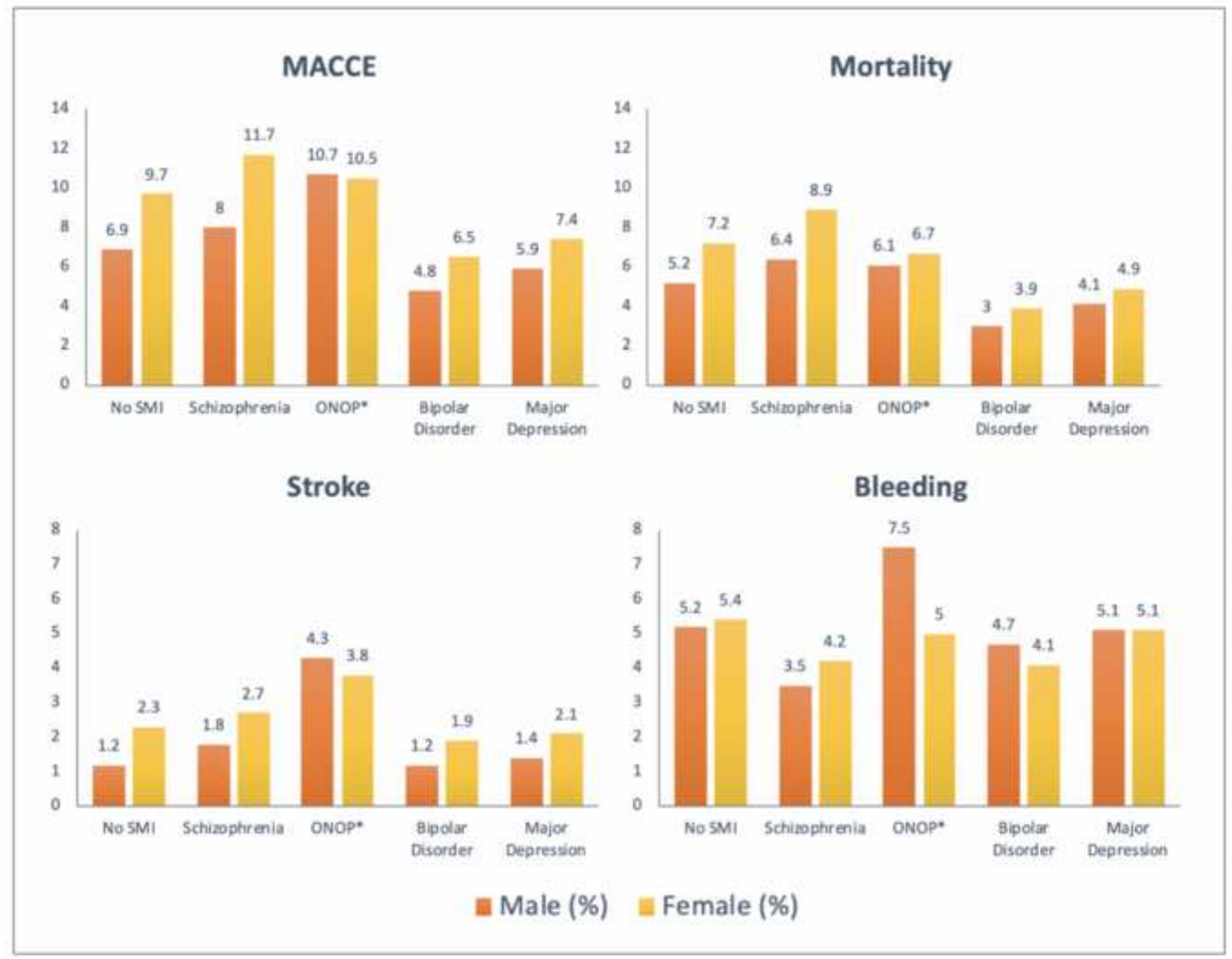




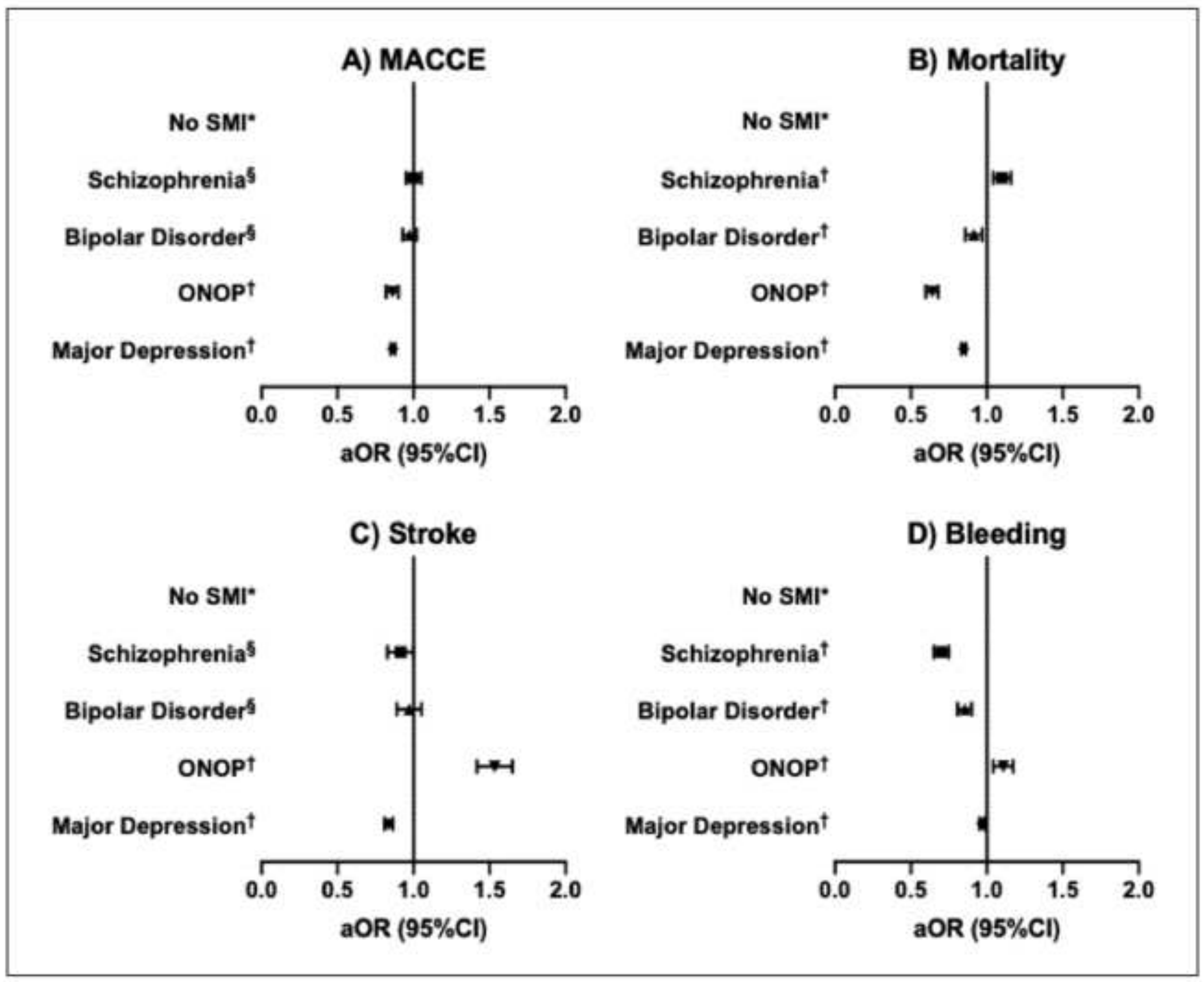


E) $\mathrm{CA}$

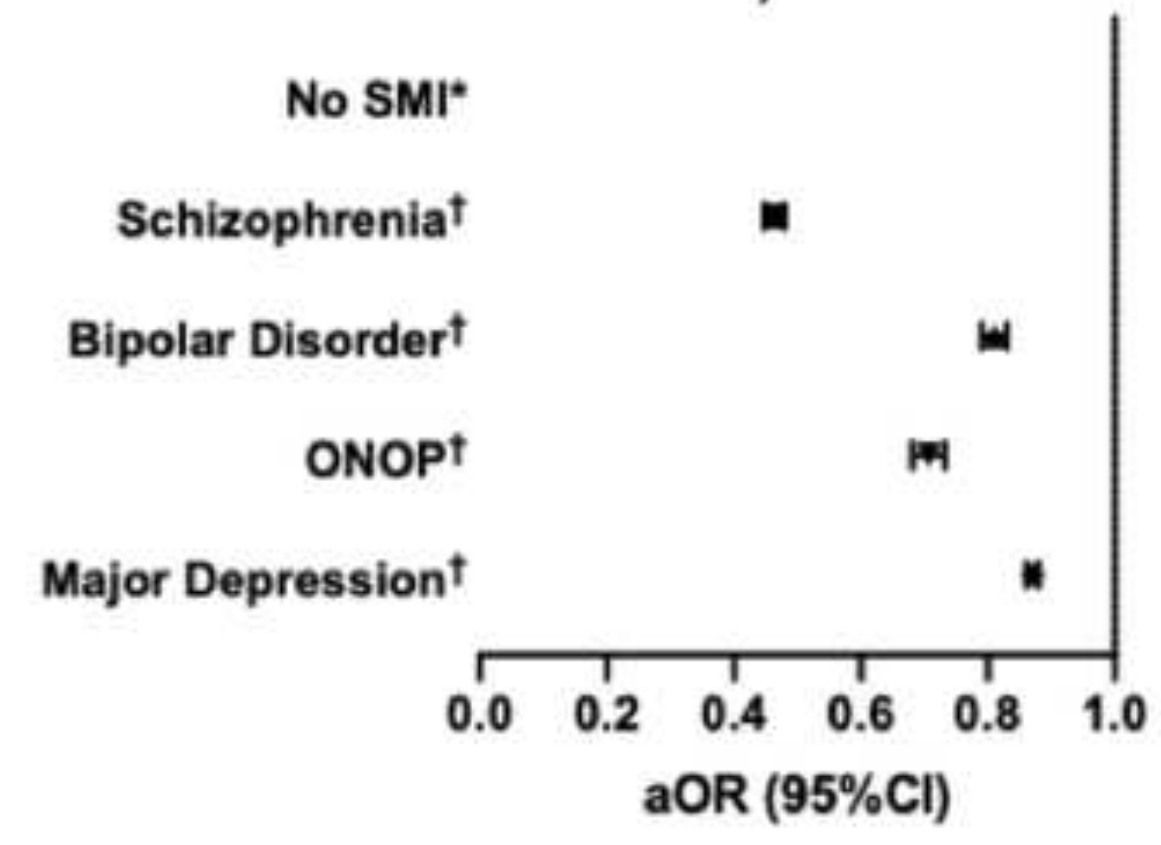

F) $\mathrm{PCl}$

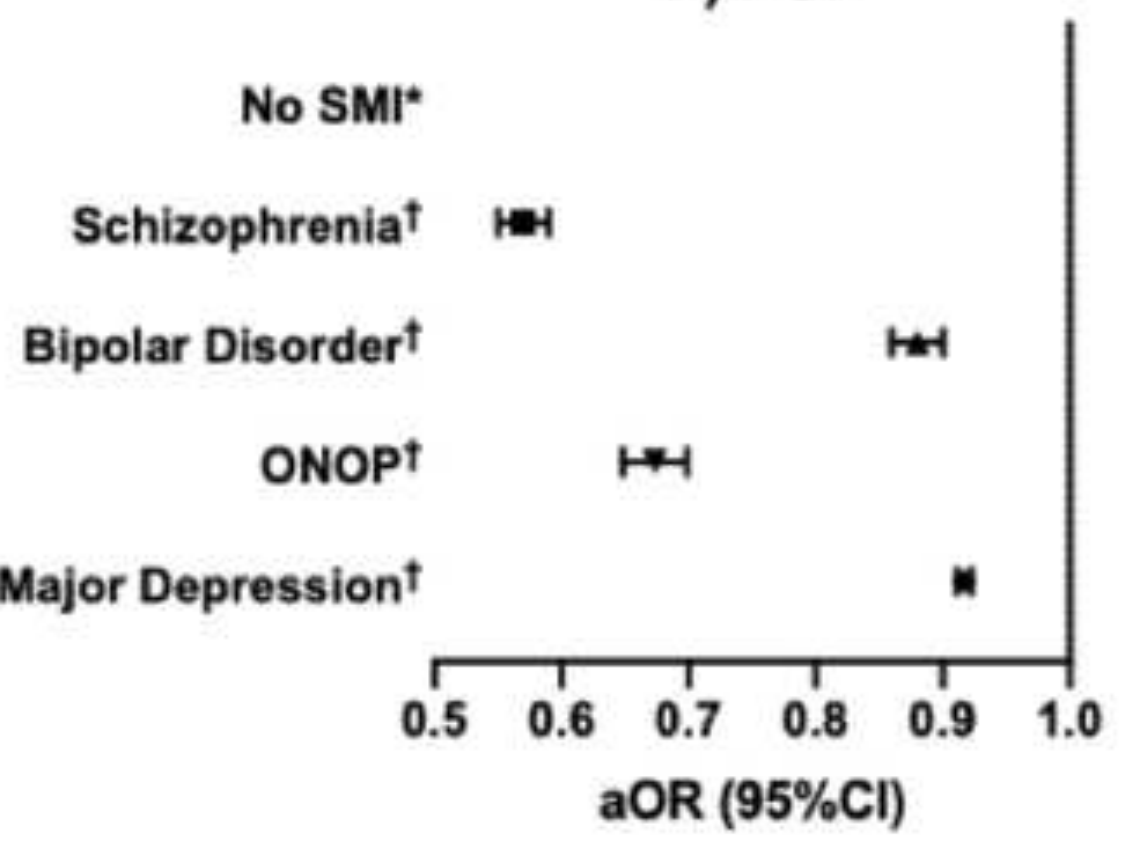



Supplementary Material
Click here to download S

Click here to download Supplementary Material: AMI Mental health outcomes Supplementary Material.docx 